\title{
Experimental Results of Novel DoA Estimation Algorithms for Compact Reconfigurable Antennas
}

\author{
Henna Paaso, ${ }^{1}$ Aki Hakkarainen, ${ }^{2}$ Nikhil Gulati, ${ }^{3}$ Damiano Patron, ${ }^{3}$ Kapil R. Dandekar, \\ Mikko Valkama, ${ }^{2}$ and Aarne Mämmelä ${ }^{1}$ \\ ${ }^{1}$ VTT Technical Research Centre of Finland, Kaitoväylä 1, P.O. Box 1100, 90571 Oulu, Finland \\ ${ }^{2}$ Department of Electronics and Communications Engineering, Tampere University of Technology, P.O. Box 553, 33101 Tampere, Finland \\ ${ }^{3}$ Department of Electrical and Computer Engineering, Drexel University, 3141 Chestnut Street, Philadelphia, PA 19104, USA
}

Correspondence should be addressed to Henna Paaso; henna.paaso@vtt.fi

Received 9 September 2016; Revised 18 December 2016; Accepted 13 April 2017; Published 24 July 2017

Academic Editor: Sergiy A. Vorobyov

Copyright (C) 2017 Henna Paaso et al. This is an open access article distributed under the Creative Commons Attribution License, which permits unrestricted use, distribution, and reproduction in any medium, provided the original work is properly cited.

\begin{abstract}
Reconfigurable antenna systems have gained much attention for potential use in the next generation wireless systems. However, conventional direction-of-arrival (DoA) estimation algorithms for antenna arrays cannot be used directly in reconfigurable antennas due to different design of the antennas. In this paper, we present an adjacent pattern power ratio (APPR) algorithm for twoport composite right/left-handed (CRLH) reconfigurable leaky-wave antennas (LWAs). Additionally, we compare the performances of the APPR algorithm and LWA-based MUSIC algorithms. We study how the computational complexity and the performance of the algorithms depend on number of selected radiation patterns. In addition, we evaluate the performance of the APPR and MUSIC algorithms with numerical simulations as well as with real world indoor measurements having both line-of-sight and non-line-ofsight components. Our performance evaluations show that the DoA estimates are in a considerably good agreement with the real DoAs, especially with the APPR algorithm. In summary, the APPR and MUSIC algorithms for DoA estimation along with the planar and compact LWA layout can be a valuable solution to enhance the performance of the wireless communication in the next generation systems.
\end{abstract}

\section{Introduction}

Direction-of-arrival (DoA) estimation algorithms using compact antenna sizes are of significant importance to next generation wireless systems. Directive adaptive antennas can estimate the DoA, steer the beam to the desired direction, and suppress the power in undesired directions to avoid interference. Thus, they can greatly improve the spectrum reuse, interference avoidance, and device localization.

Adaptive antennas can be divided into two categories: phased arrays and reconfigurable antennas. Conventionally, phased arrays use many antenna array elements to adapt the radiation pattern shape and beam direction $[1$, 2]. Antenna arrays usually require one receiver chain per antenna branch. The cost usually increases with the number of antenna elements because the array needs the same number of radio frequency (RF) high-power or low-noise amplifiers as the elements in traditional antenna arrays [3].
Particularly for digital beamforming antennas, the same number of frequency converters, RF amplifiers, and digitalto-analog (D/A) or analog-to-digital $(\mathrm{A} / \mathrm{D})$ converters are needed. This leads to high-power consumption and high fabrication cost. In contrast to multielement antenna arrays, reconfigurable antennas do not need the multiple antenna elements or feeding networks [4]. In this paper, we present a certain type of compact reconfigurable antennas, namely, the composite right/left-handed (CRLH) leaky-wave antenna (LWA) [5]. The reconfigurable LWAs have many advantages: low manufacturing cost, low DC power consumption, fullspace beam scanning using significantly less printed circuit board space, and absence of extra RF circuitry. By considering these advantages, especially compactness and beamsteering, CRLH-LWAs have a significant potential to be used in DoA systems.

In the context of conventional antenna arrays, DoA estimation algorithms have been extensively researched in 


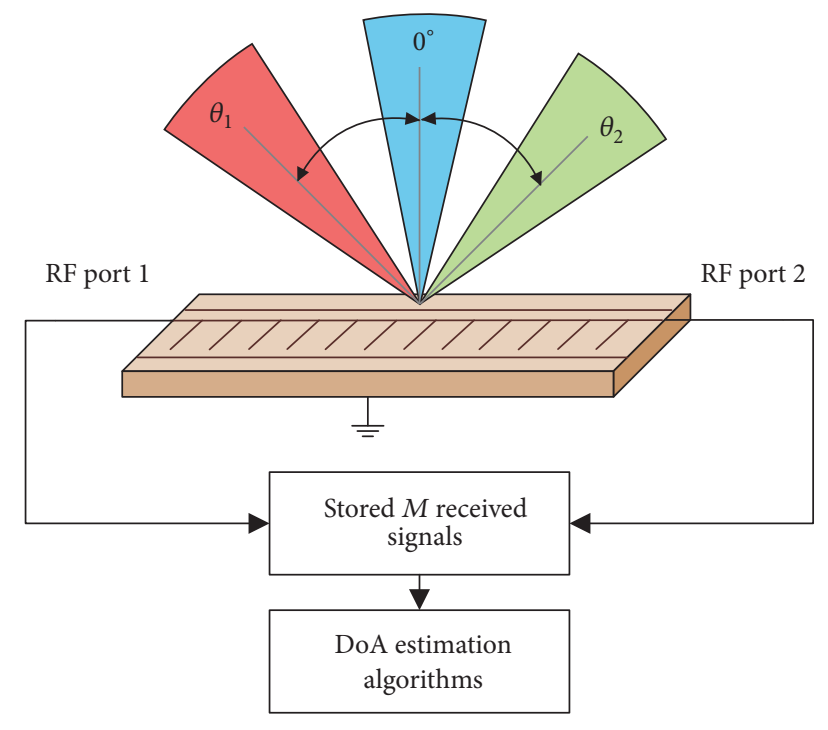

FIGURE 1: System model.

the literature [6-10]. However, these algorithms cannot be used directly in CRLH-LWAs because of the inherent design and operation of the LWAs versus multielement antenna arrays. An LWA has only one port and thus it has a single observation available at each sampling incident unlike in multielement antenna arrays where signals can be detected from different elements of the antenna array [11]. For this reason, DoA estimation algorithms for LWAs require that the LWA receives the transmitted signal $M$ times through $M$ different radiation patterns, as illustrated in Figure 1.

There are only a limited number of papers where DoA estimation algorithms are introduced for LWAs. In [12, 13], we preliminarily introduced a modified multiple signal classification (MUSIC) algorithm for LWAs. The CRLH-LWA has the inherent difference in the design. Our modified MUSIC algorithm uses $M$ received signals, each obtained with a different radiation pattern and measured from two antenna ports. Then, a correlation matrix can be formed by using these $M$ received signals. We presented experimental results of a LWA-based MUSIC algorithms in [14] where we introduced the performance of the MUSIC and power pattern crosscorrelation (PPCC) algorithms in real wireless multipath environment. However, the paper presents only results for one radiation pattern configuration and does not study how different configurations affect the performance of the algorithms. Additionally, an adjacent pattern power ratio (APPR) algorithm and the complexity analysis of the algorithms are not presented in [14]. Similar work for DoA estimation has also been presented independently in $[15,16]$ that use the MUSIC algorithm for CRLH-LWA. In [15], only experimental results are introduced and the authors do not present how the MUSIC algorithm can be built up using LWAs. Additionally, $[15,16]$ present only experimental results of the MUSIC algorithm in an anechoic chamber. In addition, $[17,18]$ introduce reactance domain MUSIC [17] and unitary MUSIC [18] algorithms for electronically steerable parasitic array radiator (ESPAR) antennas. In [19], APPR algorithm is introduced for the ESPAR antenna. The APPR algorithm is a simple DoA estimation algorithm which measures signal powers from $M$ different directions and chooses the radiation pattern with the maximum signal power. Then, the adjacent pattern power ratios with respect to the maximum signal power pattern are calculated and the DoA is estimated by comparing these ratios with the predefined ratios from a look-up table (LUT). The performance of the algorithm is presented by using computer simulations and experiments in an anechoic chamber.

In this paper, we apply the APPR DoA estimation algorithm for CRLH-LWA. Additionally, we compare the performance of the APPR algorithm with the performance of the LWA-based MUSIC algorithm. The computational complexity of the APPR algorithm is much smaller than that of the MUSIC algorithm without much degradation in estimation accuracy. Unlike in our previous studies [12-14] and other DoA estimation techniques in the literature [19], we now study how the computational complexity and the performance of the algorithms depend on the number of selected radiation patterns in different kinds of environments: theoretical additive white Gaussian noise (AWGN) channel and a real world indoor multipath wireless environment with both line-of-sight (LoS) and non-line-of-sight (NLoS) components.

This paper is organized as follows: Section 2 introduces a detailed description of the DoA estimation algorithms for a compact CRLH-LWA. Section 3 presents shortly the CRLH-LWA design. Numerical and complexity analysis and experimental results of the DoA estimation algorithms are presented in Section 4. Finally, conclusions are drawn in Section 5.

\section{CRLH-LWA-Based DoA Estimation Algorithms}

In this section, we introduce an APPR DoA estimation algorithm for LWAs. Additionally, we present briefly a modified single/two-port MUSIC algorithms [12] whose performance we compare with the APPR algorithm. The first algorithm is based on evaluating the received powers for different voltage sets and the second algorithm is variant of the popular MUSIC algorithm [6] that we have modified to work with LWA. In the following sections, $\theta$ denotes the DoA and $\widehat{\theta}$ an estimate thereof.

2.1. Single/Two-Port MUSIC Algorithm. Traditionally, the MUSIC algorithm [6] defines the spatial correlation matrix using the signals received by multiple antenna elements. In this subsection, we present how the spatial capabilities of the traditional antenna array can be virtually formulated with the CRLH-LWA and how the correlation matrix can be defined when using the two-port LWA.

Firstly, we have to collect the same transmitted signal $u(k), M$ times, while the LWA switches between $M$ different radiation patterns. Thus, we can recreate spatial diversity due to $M$ observations of the same signal but with different 
beams. With the LWA, the $M \times 1$ received signal vector $\mathbf{y}(k)$ can be expressed as

$$
\mathbf{y}(k)=\mathbf{a}(\theta) u(k)+\mathbf{z}(k),
$$

where $k$ denotes the received symbol index, $\mathbf{z}(k)$ is an $M \times 1$ AWGN vector, where the elements have variance equal to $\sigma^{2}$, and $\mathbf{a}(\theta)$ is the $M \times 1$ steering vector. The $m$ th element of the steering vector can be defined as

$$
\begin{aligned}
& a_{m}(\theta) \\
& \quad=\sqrt{G_{m}^{\prime}} \sum_{n=1}^{N} I_{n} \exp \left[j(n-1) k_{0} d\left(\sin (\theta)-\sin \left(\theta_{m}\right)\right)\right],
\end{aligned}
$$

where $m=1, \ldots, M, N$ is the number of cascaded unit cells, and

$$
G_{m}^{\prime}=\frac{G_{m}}{\left(\sum_{n=1}^{N} I_{n}\right)^{2}},
$$

where $\theta$ is the angular direction of the received signal, $\theta_{m}$ is the main beam direction of the LWA with control voltage set $m, I_{n}=I_{0} \exp [-\alpha(n-1) d]$ is an exponential function with a leakage factor $\alpha$, and $G_{m}$ is the measured antenna gain (in linear scale) of set $m$. The initial value of the exponential function $I_{0}=1$, with structure period $d$ [5].

The MUSIC algorithm makes eigenvalue decomposition of the signal covariance matrix and uses a subspace algorithm to estimate DoA. Firstly, we have to estimate the covariance matrix from the received signals. The estimated covariance matrix can be expressed as

$$
\widehat{\mathbf{R}}_{y y}=\frac{1}{N_{s}} \sum_{k=1}^{N_{s}} \mathbf{y}(k) \mathbf{y}^{\dagger}(k),
$$

where $N_{\mathrm{s}}$ denotes the number of samples and the Hermitian transpose of $\mathbf{y}$ is denoted as $\mathbf{y}^{\dagger}$. Then, the eigenvalue decomposition (EVD) of $\widehat{\mathbf{R}}_{y y}$ can be represented as

$$
\widehat{\mathbf{R}}_{y y}=\widehat{\mathbf{E}}_{s} \widehat{\Lambda}_{s} \widehat{\mathbf{E}}_{s}^{\dagger}+\widehat{\mathbf{E}}_{n} \widehat{\Lambda}_{n} \widehat{\mathbf{E}}_{n}^{\dagger},
$$

where $\widehat{\mathbf{E}}_{s}=\left[\widehat{\mathbf{e}}_{1}, \widehat{\mathbf{e}}_{2}, \ldots, \widehat{\mathbf{e}}_{L}\right]$ includes the estimated eigenvectors for the signal subspace, $\widehat{\Lambda}_{s}=\operatorname{diag}\left[\widehat{\lambda}_{1}, \widehat{\lambda}_{2}, \ldots, \widehat{\lambda}_{L}\right]$ denotes a diagonal matrix of the largest estimated eigenvalues, and $L$ is the number of incident sources. Additionally, $\widehat{\mathbf{E}}_{n}=\left[\widehat{\mathbf{e}}_{L+1}, \widehat{\mathbf{e}}_{L+2}, \ldots, \widehat{\mathbf{e}}_{M}\right]$ is the noise subspace matrix and $\widehat{\Lambda}_{n}=\operatorname{diag}\left[\widehat{\lambda}_{L+1}, \widehat{\lambda}_{L+2}, \ldots, \widehat{\lambda}_{M}\right]$ denotes the diagonal matrix of $M-L$ noise eigenvalues. Finally, the MUSIC pseudospectrum can be generated as

$$
P_{\text {MUSIC }}(\theta)=\frac{\mathbf{a}^{\dagger}(\theta) \mathbf{a}(\theta)}{\mathbf{a}^{\dagger}(\theta) \widehat{\mathbf{E}}_{n} \widehat{\mathbf{E}}_{n}^{\dagger} \mathbf{a}(\theta)} .
$$

The estimated direction-of-arrival (DoA) is the angle where the pseudospectrum $P_{\text {MUSIC }}(\theta)$ attains its maximum; that is,

$$
\widehat{\theta}_{\text {MUSIC }}=\underset{\theta}{\arg \max } P_{\text {MUSIC }}(\theta) .
$$

The MUSIC algorithm is limited to uncorrelated signals. The estimated covariance matrix $\widehat{\mathbf{R}}_{y y}$ is nonsingular as long as the incident signals are not highly correlated [20]. When the incident signals are highly correlated signals or signals with a low SNR, the performance of the MUSIC algorithms reduces or the algorithm fails even completely [21]. Thus, the MUSIC algorithm has problems when determining the number of impinging source; that is, it cannot divide the signal subspace and noise subspace correctly, and thus it is not able to estimate the spatial spectrum correctly. This problem can be solved by using spatial smoothing techniques, signal feature vector technique, and frequency smoothing techniques, among others. However, these techniques are out of the scope of this paper.

2.2. Adjacent Pattern Power Ratio. Adjacent pattern power ratio is introduced for ESPAR antennas in [19]. The APPR algorithm calculates the adjacent power pattern ratio between the maximum received power pattern and the adjacent patterns. In this paper, we show how to apply the APPR algorithm to LWAs and show how configuring the antenna radiation patterns for signal observations can impact the performance of the algorithm, which has not been further evaluated in [19]. We first calculate the received power $P_{m}$ from $M$ different directions and normalize each power yielding the gain-normalized powers $P_{m}^{\text {norm }}=P_{m} / G_{m}$. Then, the radiation pattern that gives the maximum received power is selected. Thereafter, the adjacent pattern power ratio between the adjacent pattern to the selected pattern is calculated. Firstly, the APPR ratio is calculated from the measured radiation patterns in an anechoic chamber. The APPR can be presented as

$$
\begin{gathered}
\Gamma_{m+}(\theta)=\frac{P_{m}^{\text {norm }}(\theta)}{P_{m+1}^{\text {norm }}(\theta)}, \\
\Gamma_{m-}(\theta)=\frac{P_{m}^{\text {norm }}(\theta)}{P_{m-1}^{\text {norm }}(\theta)} .
\end{gathered}
$$

Equation (8) tells that when the DoA falls into the right side of the $m$ th pattern, $P_{m+1}^{\text {norm }}(\theta)>P_{m-1}^{\text {norm }}(\theta)$, as illustrated in Figure 2. Additionally, (9) tells that when the DoA falls into the left side of the $m$ th pattern, $P_{m-1}^{\text {norm }}(\theta)>P_{m+1}^{\text {norm }}(\theta)$. These ratios for the selected $\theta$ range are calculated and stored into an LUT beforehand to reduce run-time computations. Hence, the DoA is to be estimated over the $\theta$ range. The length of $\theta$ range, $J$, depends on how radiation pattern configuration is selected. These searching areas are illustrated as a dotted line in Figure 2. Secondly, the APPR is measured for the received power and it can be presented as

$$
\begin{aligned}
\widehat{\Gamma}_{m+}(\theta) & =\frac{\widehat{P}_{m}^{\text {norm }}(\theta)}{\widehat{P}_{m+1}^{\text {norm }}(\theta)}, \\
\widehat{\Gamma}_{m-}(\theta) & =\frac{\widehat{P}_{m}^{\text {norm }}(\theta)}{\widehat{P}_{m-1}^{\text {norm }}(\theta)} .
\end{aligned}
$$

Finally, we compare these adjacent pattern power ratios. The selection of $\Gamma_{m+/ m-}$ is defined in the following way: if 


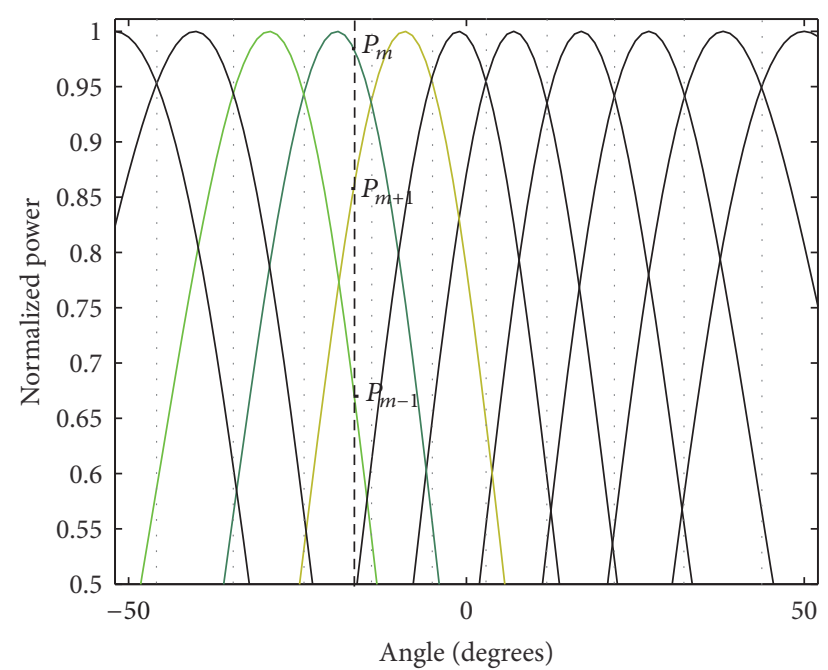

FIGURE 2: Theoretical radiation patterns.

$\widehat{P}_{m+1}^{\text {norm }}(\theta)>\widehat{P}_{m-1}^{\text {norm }}(\theta)$, we compare $\Gamma_{m+}(\theta)$ and $\widehat{\Gamma}_{m+}(\theta)$. If $\widehat{P}_{m+1}^{\text {norm }}(\theta)<\widehat{P}_{m-1}^{\text {norm }}(\theta)$, we compare $\Gamma_{m-}(\theta)$ and $\widehat{\Gamma}_{m-}(\theta)$. Hence, the estimated DoA can be defined as

$\widehat{\theta}$

$$
= \begin{cases}\arg \min \left|\Gamma_{m-}(\theta)-\widehat{\Gamma}_{m-}(\theta)\right| & \text { if } \widehat{P}_{m+1}^{\text {norm }}(\theta)<\widehat{P}_{m-1}^{\text {norm }}(\theta), \\ \arg \min \left|\Gamma_{m+}(\theta)-\widehat{\Gamma}_{m+}(\theta)\right| & \text { if } \widehat{P}_{m+1}^{\text {norm }}(\theta)>\widehat{P}_{m-1}^{\text {norm }}(\theta) .\end{cases}
$$

The computational complexity and the performance of the algorithm depend on the number of received directions. These options are researched in Section 4.

\section{Antenna Design}

The APPR and MUSIC algorithms were designed to perform the DoA estimation using reconfigurable CRLH-LWA. The leaky-wave antenna is a traveling-wave antenna [5]. As opposed to conventional resonating-wave antennas, LWAs leak out energy progressively as the wave travels along the waveguide structure. The main radiation beam of the LWA is normal to the plane of the antenna and can be in general directed by changing the electrical properties of the radiating elements.

The introduced LWA consists of 12 cascaded metamaterial CRLH unit cells which are populated with two varactor diodes in series and one in shunt configuration. The physical size of the antenna is $156 \mathrm{~mm}-38 \mathrm{~mm}$. By changing the two DC bias voltages across the varactors, the antenna is able to steer the main beam from broadside to backward and forward directions. Due to the practically symmetric antenna structure, the ports of the antenna have symmetric radiation properties with respect to the broadside direction. The beam symmetry is illustrated in Figure 1 where $\theta_{1}=-\theta_{2}$. The used LWAs are able to steer their main beam orientation approximately from $-50^{\circ}$ to $+50^{\circ}$. The CRLH-LWAs were adjusted to operate within the entire $2.4 \mathrm{GHz}$ WiFi band. The measurements of the radiation patterns were measured at the
TABLE 1: Measured LWA main beam directions and gains.

\begin{tabular}{lcccccc}
\hline Sector & 1 & 2 & 3 & 4 & 5 & 6 \\
\hline Main beam direction $\theta_{m}\left({ }^{\circ}\right)$ & \pm 0 & \pm 8 & \pm 18 & \pm 28 & \pm 39 & \pm 47 \\
Gain $G_{m}(\mathrm{~dB})$ & 5.0 & 5.1 & 5.6 & 5.8 & 4.9 & 3.5 \\
\hline
\end{tabular}

TABLE 2: Selection of radiation patterns in DoA estimation cases.

\begin{tabular}{lccccccccccc}
\hline \multirow{2}{*}{ Case } & \multicolumn{110}{c}{ Main beam directions $\left(^{\circ}\right.$ ) } & & \\
& -47 & -39 & -28 & -18 & -8 & 0 & 8 & 18 & 28 & 39 & 47 \\
\hline Case 1 & $\mathrm{x}$ & $\mathrm{x}$ & $\mathrm{x}$ & $\mathrm{x}$ & $\mathrm{x}$ & $\mathrm{x}$ & $\mathrm{x}$ & $\mathrm{x}$ & $\mathrm{x}$ & $\mathrm{x}$ & $\mathrm{x}$ \\
Case 2 & $\mathrm{x}$ & & $\mathrm{x}$ & & $\mathrm{x}$ & & $\mathrm{x}$ & & $\mathrm{x}$ & & $\mathrm{x}$ \\
Case 3 & & $\mathrm{x}$ & & $\mathrm{x}$ & & $\mathrm{x}$ & & $\mathrm{x}$ & & $\mathrm{x}$ & \\
Case 4 & $\mathrm{x}$ & & & $\mathrm{x}$ & & & $\mathrm{x}$ & & & $\mathrm{x}$ & \\
Case 5 & & $\mathrm{x}$ & & & & $\mathrm{x}$ & & & & $\mathrm{x}$ & \\
\hline
\end{tabular}

frequency of $2.46 \mathrm{GHz}$ in the anechoic chamber facility. The measured main beam directions and the corresponding gains are presented in Table 1.

\section{Numerical and Complexity Analysis and Experimental Results of DoA Estimation Algorithms}

To verify and validate the performance of the proposed DoA estimation algorithms for the LWA, we perform several simulations to numerically evaluate the performances and conduct experimental measurements, which are performed in an indoor multipath environment. We research the effect of the number of radiation patterns on the accuracy of the estimated DoAs. We particularly consider different choices of the radiation patterns for estimating the DoA. In Table 2, different radiation pattern choices are shown. The first column in this table shows cases 1 to 5 that correspond to different choices for the number of radiation patterns and their main beam directions. For each case, we estimate the DoA using only the radiation patterns which are identified by crossmarks.

4.1. Simulation Setup and Numerical Analysis. Firstly, we have studied how the selection of the radiation pattern impacts on the performance of the DoA estimation algorithms. In the simulations, we formulate the received signal according to the signal model (1). We generate orthogonal frequencydivision multiplexing (OFDM) with 48 active subcarriers as the physical signal waveform. We use the same radiation patterns as in our experimental measurements. The LWA is chosen to have 6 radiation patterns with the 12 main beam directions, as shown in Table 1. In all estimation cases, 100 complex in-phase/quadrature (I/Q) samples are used to estimate the DoA. The estimations are done prior to fast Fourier transform (FFT) processing. The direction of the incoming signal is steered from $-54^{\circ}$ to $54^{\circ}$ with a resolution of $1^{\circ}$. The estimation results are averaged over 1000 signal realizations for each estimated DoA.

In Figure 3, the root-mean-squared error (RMSE) of the MUSIC and APPR algorithms as a function of the SNR is 


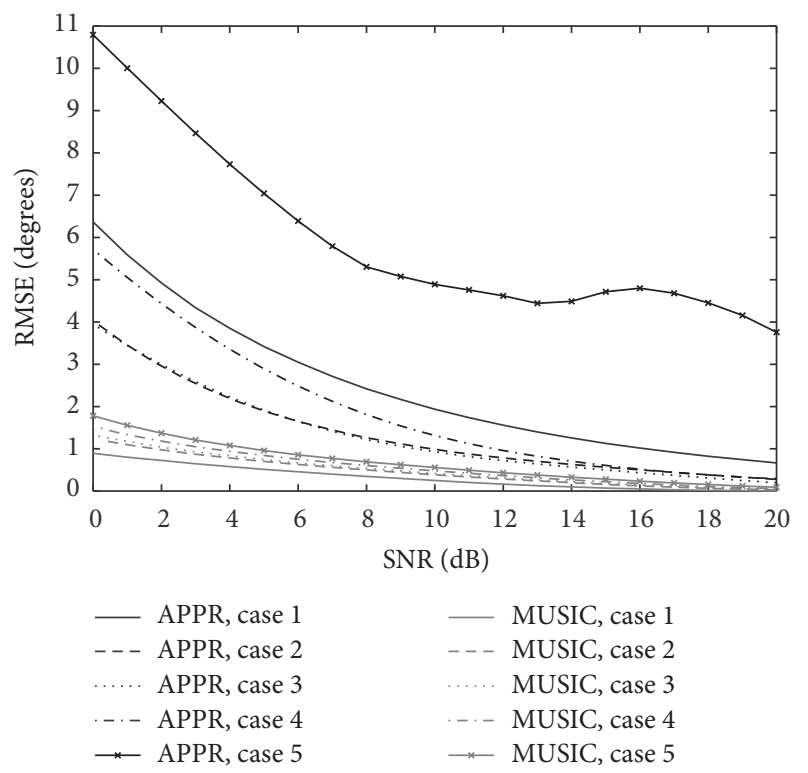

FIGURE 3: RMSE of the APPR and MUSIC algorithms as a function of the SNR.

presented. The plot shows results for cases 1-5. From Figure 3, it can be noted that the MUSIC algorithm works better than the APPR algorithm. However, if the SNR is higher than $10 \mathrm{~dB}$, the RMSE difference between the algorithms in case 3 is lower than $0.5^{\circ}$. It can be observed that case 1 gives clearly the best result for the MUSIC algorithm. Furthermore, the MUSIC algorithm has less performance difference between different cases than the APPR algorithm. For example, if the SNR is higher than $10 \mathrm{~dB}$, the differences between the different cases are less than $0.3^{\circ}$ for the MUSIC algorithm. It can be also seen that the APPR algorithm works reasonably well in all cases, except in case 5. Cases 2-3, in turn, give the best performances in all SNR values and their RMSEs are almost the same. Moreover, we can see that the RMSE is significantly large for case 5. Additionally, case 1 works worse than cases 2-4. The reason for these observations is that the radiation patterns cover very well the estimation area in cases 2-4, especially in case 3, as seen in Figure 4. The radiation patterns are not too near to or too far from each other. However, in case 1, the radiation patterns are too near, whereas they are too far in case 5 (as also visible in Figure 5), causing higher DoA estimation errors for the APPR algorithm.

Figures 6 and 7 depict the simulated performance of the APPR and MUSIC algorithm as a function of the DoA when the SNR is fixed to $10 \mathrm{~dB}$. It is visible in Figure 6 that the RMSE is the smoothest in cases 2-3. We can also notice that the configuration of the radiation pattern should be selected carefully for the APPR algorithm. If we selected five radiation patterns, as in case 3 , instead of the 11 radiation patterns, as in case 1 , the overall signal storing time would be halved. However, the estimation DoA range increases when using fewer radiation patterns because of the increased gap between the adjacent radiation patterns. However, we do not need to calculate the power of the signal so many times if fewer radiation patterns are selected. Furthermore, the amount of

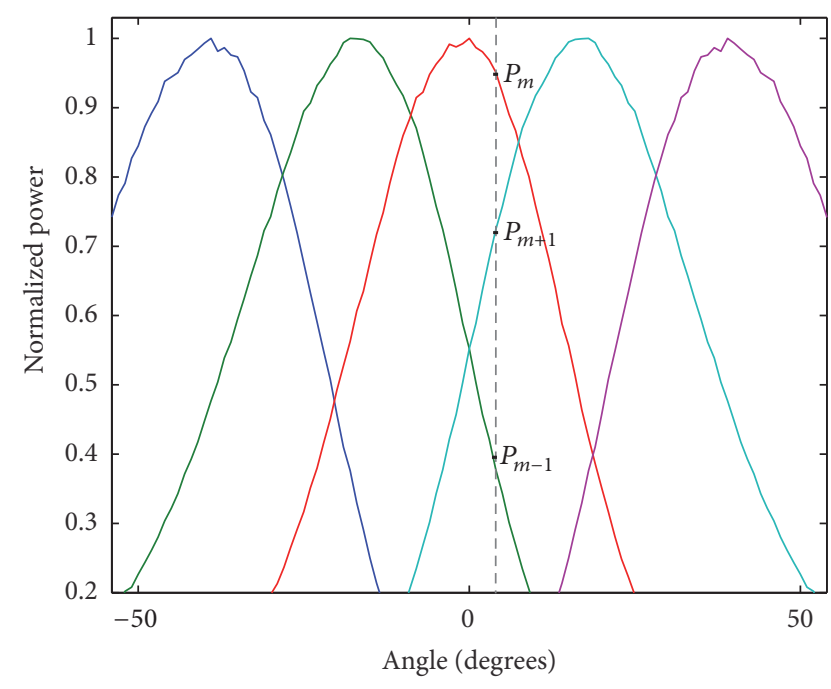

FIgURE 4: Measured radiation patterns for case 3.

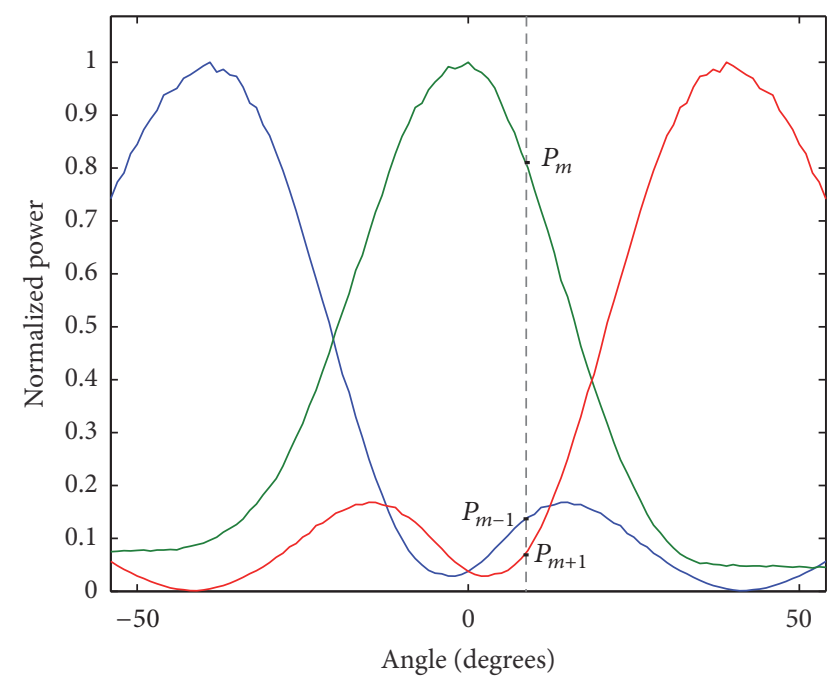

FIGURE 5: Measured radiation patterns for case 5.

data to be processed offline and online will be decreased if fewer radiation patterns are selected. Based on the results in Figure 7 for the MUSIC algorithm, we notice that the RMSE behaves similarly in all cases, and only the absolute level of the RMSE is varying in different cases. The highest number of radiation patterns provides again the best performance, as expected. From the results, we can say that the selection of the number of radiation patterns is a trade-off between the desired performance and computational complexity. In Section 4.2, computational complexity is analyzed in more detail.

4.2. Computational Complexity Analysis. In this section, we analyze the computational complexity of the single/two-port MUSIC and APPR algorithms. We define the complexity of these DoA estimation algorithms in terms of basic operations [22], that is, additions and subtractions, multiplications, and 


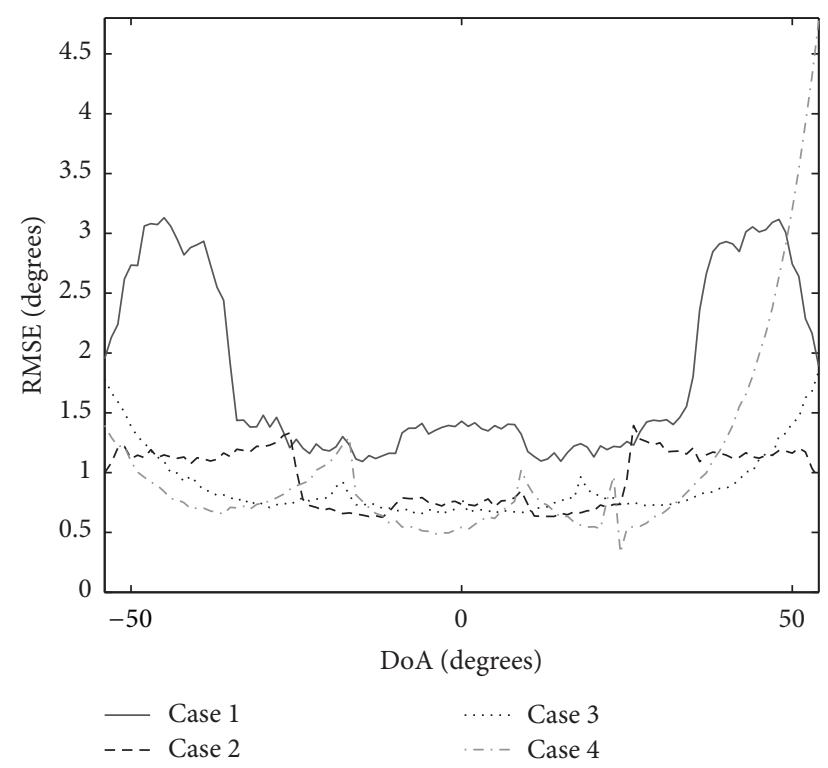

FIgURE 6: RMSE of the APPR algorithm as a function of the DoA.

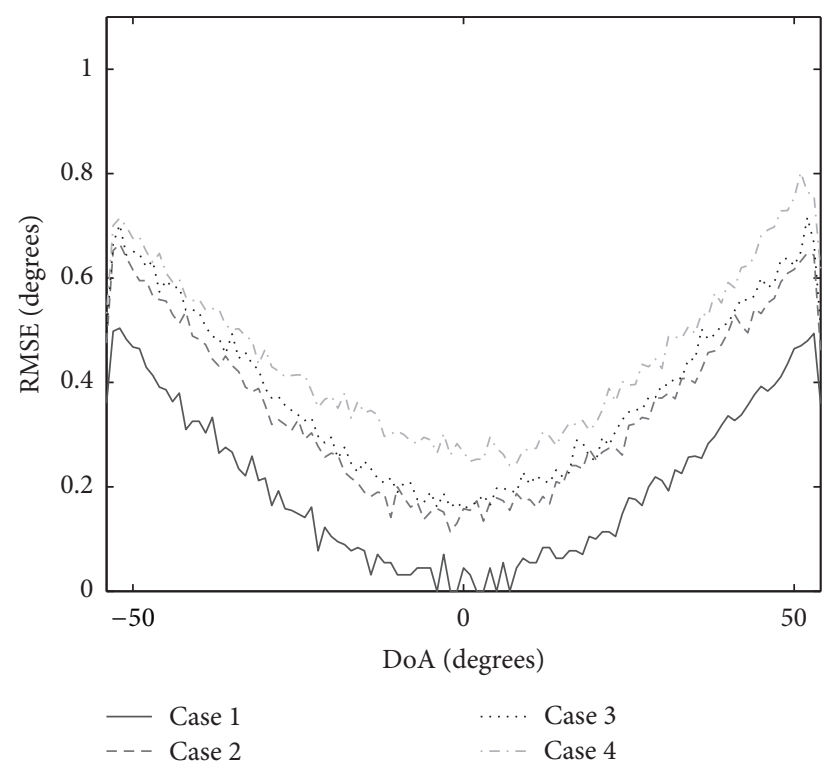

FIGURE 7: RMSE of the MUSIC algorithm as a function of the DoA.

divisions, and refer to them as ADD, MUL, and DIV, respectively. In the APPR algorithm case, part of the calculations can be calculated offline and stored into the LUT, in which case they do not need to be calculated in real time. The analysis is presented in Table 3. There, $J_{0}$ is the number of the DoA estimation points and is here equal to $109^{\circ}\left(-54^{\circ}\right.$ to $54^{\circ}$ with $1^{\circ}$ resolution). In addition, $J$ is the length of the specific range of $\theta$ for the APPR algorithm which depends on how the radiation pattern configuration is selected, as explained in Section 2.2. Selecting $M=11$ results in $J=15^{\circ}$, whereas for $M=5$ we get $J=31^{\circ}$. Regarding the computational complexities, the covariance matrix of the MUSIC algorithm has $N_{s} M^{2}$ multiplications and $\left(N_{s}-1\right) M^{2}$ additions and subtractions. Additionally, the EVD has the complexity of

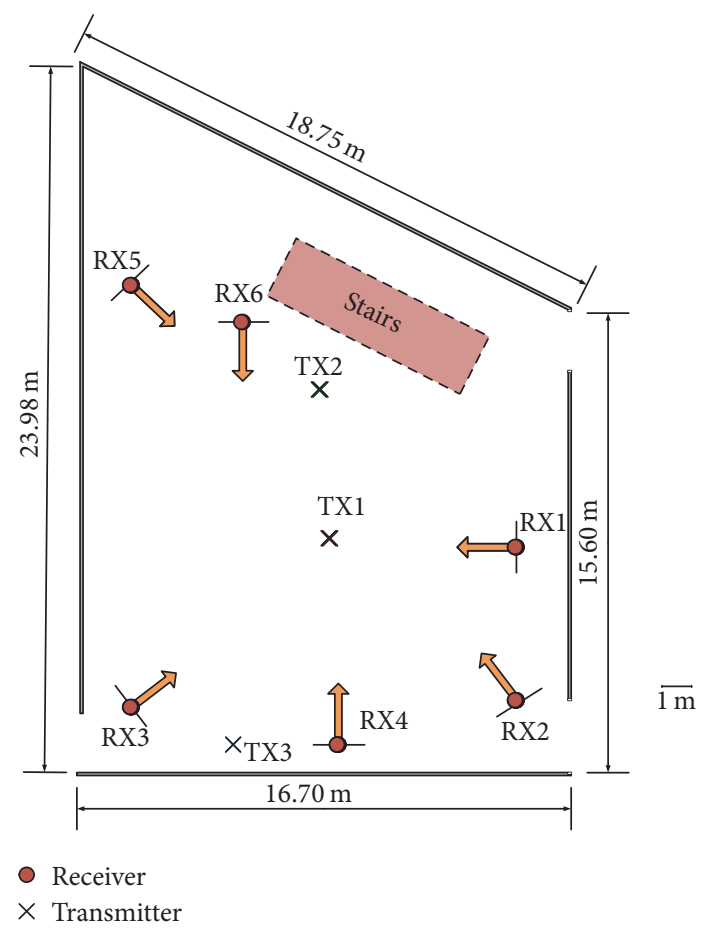

FIgURE 8: Layout of the measurement lobby area and the locations of the TXs and RXs.

TABLE 3: The computational complexity analysis.

\begin{tabular}{lcc}
\hline Algorithm & MUSIC & APPR \\
\hline MUL & $M^{3}+M^{2}\left(N_{s}+2 J_{0}\right)$ & $2 M N_{s}$ \\
DIV & $J_{0}$ & 2 \\
ADD & $M^{2}\left(N_{s}-1+2 J_{0}\right)-2 J_{0} M$ & $J+M\left(N_{s}-1\right)$ \\
LUT & - & $\left(M J_{0}\right)$ MUL \\
& & $\left(2 M J+M J_{0}\right)$ DIV \\
\hline
\end{tabular}

$M^{3}$ and the calculations of the MUSIC spectra need $J_{0} 2 M^{2}$ multiplications, $J_{0}$ divisions, and $J_{0}\left(2 M^{2}-2 M\right)$ additions and subtractions. The APPR algorithm, in turn, needs $2 M N_{s}$ multiplications, 2 divisions, and $J+M\left(N_{s}-1\right)$ additions and subtractions. In addition, we need $M J_{0}$ multiplications and $2 M J+M J_{0}$ divisions that are calculated offline and stored into a LUT. It is clear that the APPR algorithm has much lower computational complexity than the MUSIC algorithm.

4.3. Measurement Setup. The performance of the LWA-based APPR and MUSIC DoA estimation algorithms is evaluated using experimental measurements performed in a multipath indoor environment. The experiments are done in the premises of the Drexel University. The indoor setup is a closed large lobby with stairs and glass walls. In this lobby setting, the measured signals experience both LoS and NLoS components due to severe multipath between the transmitters (TXs) and the receivers (RXs). The layout, illustrated in Figure 8, shows the arrangement of TXs and RXs in the described indoor environment. In our measurements, we used three TX nodes and six RX nodes. 
In the RX nodes, the reconfigurable two-port CRLHLWAs were used and the three TX nodes were equipped with two standard omnidirectional antennas. The lobby dimensions and the locations of the transceiver antennas are carefully measured with a measuring tape. In Figure 8, the orange arrows show the broadside direction $\theta=0^{\circ}$ of the RX LWAs. We made measurements in such a way that only one TX-RX pair was active at a time. Thus, we needed to ensure a justness between different transmission links by transmitting the same data over all links. We measured all the TX-RX pairs but the data with TX3 were studied only for RX1-RX3 and RX5-RX6 because the TX3-RX4 pair was out of the spatial scanning directions.

In our measurement campaign, each transceiver uses a field-programmable gate array (FPGA) based software defined radio platform which is called wireless open-access research platform (WARP) v3 [23]. Each WARP board was connected to its own antenna and to a centralized controlling system which centrally synchronize all the nodes, control antenna beam directions, and collect all the measurement data. We used OFDM signals with the total of 64 subcarriers where 48 subcarriers were used for loading data symbols, 4 for carrier frequency offset (CFO) correction, and 12 empty subcarriers. After each transmission, all the RXs stored 300 packets, each containing 5420 binary phase shift keying (BPSK) complex symbols and then the antenna beam direction was steered for the next reception. The TX power of the TX nodes was set to $15 \mathrm{dBm}$. Additionally, we used $d=1.3 \mathrm{~cm}$ and $\alpha=1$ in the measurement processing for the MUSIC algorithm. Furthermore, we assume that only one signal is received in the RX, and thus we set $L=1$.

All the measured data were saved for offline postprocessing with the DoA estimation algorithms introduced in Section 2. The DoA estimation was done before the FFT. Furthermore, the measurements were carried out in the WiFi frequency range of $2.452 \mathrm{GHz}-2.472 \mathrm{GHz}$ as the leaky-wave antennas (LWAs) were calibrated for this range. Since the measurements were carried out at open WiFi frequencies with various $\mathrm{WiFi}$ access points in the close vicinity, all WiFi traffic acts directly as cochannel interference making the measurement environment very challenging. Additionally, this WiFi traffic and passersby are not stationary during the measurements.

4.4. Experimental Results Based on Measurements. The aim of our experiment measurement is to demonstrate the DoA estimation capabilities for LWAs using the algorithms which were introduced in Section 2. Based on the simulation results with the AWGN channel, cases 2-3 give the best RMSE results for the APPR algorithm and case 1 gives the best results for the MUSIC algorithm, and thus we analyzed these three cases also in a real multipath environment. Here, we present also the results of the power detector (PD) algorithm, as a reference because the APPR algorithm is based on these PD results. The summary of the results for the APPR, MUSIC algorithms, and PD is presented in Tables 4-12. DoA estimation results for TX1-RX6, TX3-RX6, and TX2-RX6 pairs are
TABLE 4: DoA estimation results for TX1 with PD, APPR, and MUSIC algorithms in case 1.

\begin{tabular}{lcccccc}
\hline \multirow{2}{*}{$\theta_{\text {real }}\left(^{\circ}\right)$} & $\hat{\theta}$ PD & \multicolumn{2}{c}{ APPR } & \multicolumn{2}{c}{ MUSIC } \\
& $\left(^{\circ}\right)$ & $\epsilon\left(^{\circ}\right)$ & $\hat{\theta}\left({ }^{\circ}\right)$ & $\epsilon\left(^{\circ}\right)$ & $\hat{\theta}\left({ }^{\circ}\right)$ & $\epsilon\left(^{\circ}\right)$ \\
\hline RX1: -3 & 8 & $\mathbf{1 1}$ & 12 & $\mathbf{1 5}$ & 25 & $\mathbf{2 8}$ \\
RX2: 12 & -18 & $-\mathbf{3 0}$ & -21 & $-\mathbf{3 3}$ & -16 & $\mathbf{- 2 8}$ \\
RX3: 3 & 8 & $\mathbf{5}$ & 8 & $\mathbf{5}$ & 0 & $-\mathbf{3}$ \\
RX4: 2 & -8 & $\mathbf{1 0}$ & -13 & $-\mathbf{1 5}$ & 0 & $-\mathbf{4}$ \\
RX5: -9 & -28 & $-\mathbf{1 9}$ & -33 & $-\mathbf{2 4}$ & 1 & $\mathbf{1 0}$ \\
RX6: 22 & 28 & $\mathbf{6}$ & 22 & $\mathbf{0}$ & 28 & $\mathbf{6}$ \\
RMSE & & $\mathbf{1 6 . 0}$ & & $\mathbf{1 8 . 9}$ & & $\mathbf{1 6 . 8}$ \\
\hline
\end{tabular}

TABLE 5: DoA estimation results for TX2 with PD, APPR, and MUSIC algorithms in case 1.

\begin{tabular}{lcccccc}
\hline \multirow{2}{*}{$\theta_{\text {real }}\left(^{\circ}\right)$} & \multicolumn{2}{c}{ PD } & \multicolumn{2}{c}{ APPR } & \multicolumn{2}{c}{ MUSIC } \\
& $\hat{\theta}\left(^{\circ}\right)$ & $\epsilon\left(^{\circ}\right)$ & $\hat{\theta}\left({ }^{\circ}\right)$ & $\epsilon\left(^{\circ}\right)$ & $\hat{\theta}\left({ }^{\circ}\right)$ & $\epsilon\left(^{\circ}\right)$ \\
\hline RX1: -39 & -47 & $-\mathbf{8}$ & -41 & $-\mathbf{2}$ & -54 & $-\mathbf{1 5}$ \\
RX2: -5 & -28 & $-\mathbf{2 3}$ & -24 & $-\mathbf{1 9}$ & -1 & $-\mathbf{4}$ \\
RX3: 22 & 0 & $-\mathbf{2 2}$ & 4 & $-\mathbf{1 8}$ & 18 & $-\mathbf{4}$ \\
RX4: 3 & 8 & $\mathbf{5}$ & 6 & $\mathbf{3}$ & 0 & $-\mathbf{3}$ \\
RX5: 14 & 8 & $-\mathbf{6}$ & 8 & $-\mathbf{6}$ & 1 & $-\mathbf{1 3}$ \\
RX6: 49 & 39 & $-\mathbf{1 0}$ & 39 & $-\mathbf{1 0}$ & 54 & $\mathbf{5}$ \\
RMSE & & $\mathbf{1 4 . 4}$ & & $\mathbf{1 1 . 8}$ & & $\mathbf{8 . 7}$ \\
\hline
\end{tabular}

TABLE 6: DoA estimation results for TX3 with PD, APPR, and MUSIC algorithms in case 1.

\begin{tabular}{lcccccc}
\hline \multirow{2}{*}{$\theta_{\text {real }}\left({ }^{\circ}\right)$} & \multicolumn{2}{c}{$\mathrm{PD}$} & \multicolumn{2}{c}{ APPR } & \multicolumn{2}{c}{ MUSIC } \\
& $\hat{\theta}\left({ }^{\circ}\right)$ & $\epsilon\left(^{\circ}\right)$ & $\hat{\theta}\left({ }^{\circ}\right)$ & $\epsilon\left(^{\circ}\right)$ & $\hat{\theta}\left({ }^{\circ}\right)$ & $\epsilon\left(^{\circ}\right)$ \\
\hline RX1: 35 & 47 & $\mathbf{1 2}$ & 42 & $\mathbf{7}$ & 48 & $\mathbf{1 3}$ \\
RX2: 62 & 47 & $-\mathbf{1 5}$ & 41 & $-\mathbf{2 1}$ & 54 & $\mathbf{- 8}$ \\
RX3: -57 & -47 & $\mathbf{1 0}$ & -47 & $\mathbf{1 0}$ & -54 & $\mathbf{3}$ \\
RX5: -34 & -47 & $-\mathbf{1 3}$ & -44 & $-\mathbf{1 0}$ & -54 & $\mathbf{- 2 0}$ \\
RX6: -1 & -28 & $-\mathbf{2 7}$ & -32 & $-\mathbf{3 1}$ & -17 & $-\mathbf{1 6}$ \\
RMSE & & $\mathbf{1 6 . 5}$ & & $\mathbf{1 8 . 1}$ & & $\mathbf{1 3 . 4}$ \\
\hline
\end{tabular}

also illustrated in Figures 9-11 for three considered cases. In the figures, the first column (a) illustrates a measurement case when all methods estimate very well the DoA of the received signal. The second column (b) presents a measurement when the APPR algorithm and PD fail to estimate the DoA. The last column (c) illustrates the measurement case when the APPR algorithm estimates very well the DoA while the MUSIC algorithm fails the DoA estimation.

4.4.1. Case 1. Based on the results of Tables 4-6, the total RMSE, calculated over all TX cases, is $13.9^{\circ}$ for the PD, $9.8^{\circ}$ for the APPR algorithm, and $13.6^{\circ}$ for the MUSIC algorithm in case 1 . If the DoA estimation error is large in the PD case, the APPR algorithm cannot estimate the DoA accurately, as illustrated in Figure 9(b). It can be noticed that the APPR has the best performance if we compare overall results. As we explained earlier in Section 2.1, the MUSIC algorithm works well only for uncorrelated signals. In our experimental 
TABLE 7: DoA estimation results for TX1 with PD, APPR, and MUSIC algorithms in case 2.

\begin{tabular}{lcccccc}
\hline \multirow{2}{*}{$\theta_{\text {real }}\left(^{\circ}\right)$} & $\hat{\theta}$ & PD & \multicolumn{2}{c}{ APPR } & \multicolumn{2}{c}{ MUSIC } \\
& $\left(^{\circ}\right)$ & $\epsilon\left(^{\circ}\right)$ & $\hat{\theta}\left({ }^{\circ}\right)$ & $\epsilon\left(^{\circ}\right)$ & $\hat{\theta}\left({ }^{\circ}\right)$ & $\epsilon\left(^{\circ}\right)$ \\
\hline RX1: -3 & 8 & $\mathbf{1 1}$ & 1 & $\mathbf{4}$ & 19 & $\mathbf{2 2}$ \\
RX2: 12 & -28 & $-\mathbf{4 0}$ & -18 & $-\mathbf{3 0}$ & -4 & $-\mathbf{1 6}$ \\
RX3: 3 & 8 & $\mathbf{5}$ & 1 & $-\mathbf{2}$ & 0 & $-\mathbf{3}$ \\
RX4: 2 & -8 & $\mathbf{1 0}$ & -17 & $-\mathbf{1 9}$ & -1 & $-\mathbf{3}$ \\
RX5: -9 & -28 & $-\mathbf{1 9}$ & -19 & $\mathbf{1 0}$ & 1 & $\mathbf{1 0}$ \\
RX6: 22 & 28 & $\mathbf{6}$ & 22 & $\mathbf{0}$ & 38 & $\mathbf{1 6}$ \\
RMSE & & $\mathbf{1 9 . 3}$ & & $\mathbf{1 5 . 2}$ & & $\mathbf{1 3 . 6}$ \\
\hline
\end{tabular}

TABLE 8: DoA estimation results for TX2 with PD, APPR, and MUSIC algorithms in case 2.

\begin{tabular}{lcccccc}
\hline \multirow{2}{*}{$\theta_{\text {real }}\left(^{\circ}\right)$} & $\hat{\theta}$ & PD & \multicolumn{2}{c}{ APPR } & \multicolumn{2}{c}{ MUSIC } \\
& $\hat{\theta}\left({ }^{\circ}\right)$ & $\epsilon\left(^{\circ}\right)$ & $\hat{\theta}\left({ }^{\circ}\right)$ & $\epsilon\left(^{\circ}\right)$ & $\hat{\theta}\left({ }^{\circ}\right)$ & $\epsilon\left(^{\circ}\right)$ \\
\hline RX1: -39 & -47 & $-\mathbf{8}$ & -39 & $\mathbf{0}$ & -54 & $-\mathbf{1 5}$ \\
RX2: -5 & -28 & $-\mathbf{2 3}$ & -21 & $-\mathbf{1 6}$ & 13 & $\mathbf{1 8}$ \\
RX3: 22 & 28 & $\mathbf{6}$ & 35 & $\mathbf{1 3}$ & 36 & $\mathbf{1 4}$ \\
RX4: 3 & 8 & $\mathbf{5}$ & 3 & $\mathbf{0}$ & 0 & $-\mathbf{3}$ \\
RX5: 14 & 8 & $-\mathbf{6}$ & 17 & $\mathbf{3}$ & 1 & $-\mathbf{1 3}$ \\
RX6: 49 & 47 & $-\mathbf{2}$ & 37 & $-\mathbf{1 2}$ & 37 & $-\mathbf{1 2}$ \\
RMSE & & $\mathbf{1 0 . 8}$ & & $\mathbf{9 . 8}$ & & $\mathbf{1 3 . 3}$ \\
\hline
\end{tabular}

TABLE 9: DoA estimation results for TX3 with PD, APPR, and MUSIC algorithms in case 2.

\begin{tabular}{lcccccc}
\hline \multirow{2}{*}{$\theta_{\text {real }}\left(^{\circ}\right)$} & $\hat{2}$ PD & \multicolumn{2}{c}{ APPR } & \multicolumn{2}{c}{ MUSIC } \\
& $\hat{\theta}\left(^{\circ}\right)$ & $\epsilon\left(^{\circ}\right)$ & $\hat{\theta}\left({ }^{\circ}\right)$ & $\epsilon\left(^{\circ}\right)$ & $\hat{\theta}\left({ }^{\circ}\right)$ & $\epsilon\left(^{\circ}\right)$ \\
\hline RX1: 35 & 47 & $\mathbf{1 2}$ & 40 & $\mathbf{5}$ & 54 & $\mathbf{1 9}$ \\
RX2: 62 & 47 & $-\mathbf{1 5}$ & 38 & $-\mathbf{2 4}$ & 54 & $\mathbf{- 8}$ \\
RX3: -57 & -47 & $\mathbf{1 0}$ & -46 & $\mathbf{1 1}$ & -54 & $\mathbf{3}$ \\
RX5: -34 & -47 & $-\mathbf{1 3}$ & -42 & $-\mathbf{8}$ & -54 & $\mathbf{- 2 0}$ \\
RX6: -1 & -28 & $-\mathbf{2 7}$ & -33 & $-\mathbf{3 2}$ & -23 & $-\mathbf{2 2}$ \\
RMSE & & $\mathbf{1 6 . 5}$ & & $\mathbf{1 9 . 0}$ & & $\mathbf{1 6 . 2}$ \\
\hline
\end{tabular}

TABLE 10: DoA estimation results for TX1 with PD, APPR, and MUSIC algorithms in case 3.

\begin{tabular}{lcccccc}
\hline \multirow{2}{*}{$\theta_{\text {real }}\left(^{\circ}\right)$} & $\hat{2}$ PD & \multicolumn{2}{c}{ APPR } & \multicolumn{2}{c}{ MUSIC } \\
& $\hat{\theta}\left(^{\circ}\right)$ & $\epsilon\left(^{\circ}\right)$ & $\hat{\theta}\left({ }^{\circ}\right)$ & $\epsilon\left(^{\circ}\right)$ & $\hat{\theta}\left({ }^{\circ}\right)$ & $\epsilon\left(^{\circ}\right)$ \\
\hline RX1: -3 & -18 & $-\mathbf{1 5}$ & -8 & $-\mathbf{5}$ & -4 & $\mathbf{1}$ \\
RX2: 12 & -18 & $-\mathbf{3 0}$ & -26 & $\mathbf{3 8}$ & -13 & $-\mathbf{2 5}$ \\
RX3: 3 & 0 & $-\mathbf{3}$ & 8 & $\mathbf{5}$ & 32 & $\mathbf{2 9}$ \\
RX4: 2 & -18 & $\mathbf{1 0}$ & -9 & $-\mathbf{1 1}$ & -2 & $-\mathbf{4}$ \\
RX5: -9 & -39 & $-\mathbf{3 0}$ & -28 & $-\mathbf{1 9}$ & -19 & $-\mathbf{1 0}$ \\
RX6: 22 & 18 & $-\mathbf{4}$ & 26 & $\mathbf{4}$ & 17 & $-\mathbf{5}$ \\
RMSE & & $\mathbf{2 0 . 2}$ & & $\mathbf{1 8 . 2}$ & & $\mathbf{1 6 . 4}$ \\
\hline
\end{tabular}

measurements, the measured signals experience both LoS and NLoS components due to severe multipath between the TX and the RX in the indoor environment. Multipath signals are mutually correlated, and the signal covariance becomes rank-deficient [21]. Consequently, the eigenvalue
TABLE 11: DoA estimation results for TX2 with PD, APPR, and MUSIC algorithms in case 3.

\begin{tabular}{lcccccc}
\hline \multirow{2}{*}{$\theta_{\text {real }}\left({ }^{\circ}\right)$} & \multicolumn{2}{c}{ PD } & \multicolumn{2}{c}{ APPR } & \multicolumn{2}{c}{ MUSIC } \\
& $\hat{\theta}\left({ }^{\circ}\right)$ & $\epsilon\left(^{\circ}\right)$ & $\hat{\theta}\left({ }^{\circ}\right)$ & $\epsilon\left(^{\circ}\right)$ & $\hat{\theta}\left({ }^{\circ}\right)$ & $\epsilon\left(^{\circ}\right)$ \\
\hline RX1: -39 & -39 & $\mathbf{0}$ & -32 & $\mathbf{7}$ & -54 & $-\mathbf{1 5}$ \\
RX2: -5 & -18 & $-\mathbf{1 3}$ & -26 & $-\mathbf{2 1}$ & -3 & $\mathbf{2}$ \\
RX3: 22 & 0 & $-\mathbf{2 2}$ & 6 & $\mathbf{1 6}$ & -15 & $-\mathbf{3 7}$ \\
RX4: 3 & -18 & $\mathbf{2 1}$ & -11 & $-\mathbf{1 4}$ & -1 & $\mathbf{- 4}$ \\
RX5: 14 & 0 & $-\mathbf{1 4}$ & 9 & $-\mathbf{5}$ & 6 & $-\mathbf{8}$ \\
RX6: 49 & 39 & $\mathbf{- 1 0}$ & 32 & $-\mathbf{1 7}$ & 54 & $\mathbf{5}$ \\
RMSE & & $\mathbf{1 5 . 2}$ & & $\mathbf{1 4 . 5}$ & & $\mathbf{1 6 . 8}$ \\
\hline
\end{tabular}

TABLE 12: DoA estimation results for TX3 with PD, APPR, and MUSIC algorithms in case 3.

\begin{tabular}{lcccccc}
\hline \multirow{2}{*}{$\theta_{\text {real }}\left({ }^{\circ}\right)$} & $\hat{2}$ PD & \multicolumn{2}{c}{ APPR } & \multicolumn{2}{c}{ MUSIC } \\
& $\hat{\theta}\left({ }^{\circ}\right)$ & $\epsilon\left(^{\circ}\right)$ & $\hat{\theta}\left({ }^{\circ}\right)$ & $\epsilon\left(^{\circ}\right)$ & $\hat{\theta}\left({ }^{\circ}\right)$ & $\epsilon\left(^{\circ}\right)$ \\
\hline RX1: 35 & 39 & $\mathbf{4}$ & 35 & $\mathbf{0}$ & 54 & $\mathbf{1 9}$ \\
RX2: 62 & 39 & $-\mathbf{2 3}$ & 29 & $\mathbf{3 3}$ & 54 & $\mathbf{- 8}$ \\
RX3: -57 & -39 & $\mathbf{1 8}$ & -42 & $\mathbf{1 5}$ & 54 & $\mathbf{1 1 1}$ \\
RX5: -34 & -39 & $-\mathbf{5}$ & -34 & $-\mathbf{3 4}$ & -54 & $\mathbf{- 2 0}$ \\
RX6: -1 & -39 & $\mathbf{- 3 8}$ & -29 & $\mathbf{- 2 8}$ & -6 & $\mathbf{- 5}$ \\
RMSE & & $\mathbf{2 1 . 6}$ & & $\mathbf{2 0 . 5}$ & & $\mathbf{5 1 . 3}$ \\
\hline
\end{tabular}

decomposition of the signal covariance fails to split the signal and noise subspaces. That is the reason why the results of the MUSIC algorithms are so much poorer in indoor environments than in the AWGN channel environment. What is also interesting is that we can notice multiple peaks in the PD plots in Figures 9(b) and 9(c) and the power of the signal is noticeably lower than in Figure 9(a). The peaks are most likely affected by the multipath effects like reflections from the walls and stairs, passersby, or WiFi traffic acting directly as cochannel interference in these measurements. For the PD, it is clear that multipath or other signals result in additional peaks in the figures. In cases where the PD estimator has no high peaks in the results or there are two or more low peaks or the received signal power level is low, the MUSIC algorithm has difficulties in estimating the DoA. This somewhat flat response is, again, most probably affected by a weak LoS component as well as rich scattering environment causing multiple impinging NLoS signal paths.

There are large differences in the DoA estimation accuracy in different receiver locations. The estimated DoAs are in good agreement with the real DoAs in the several APPR algorithm cases, particularly in TX1-RX3, TX1-RX6, TX2RX1, and TX2-RX4 cases. The MUSIC algorithm has also good accuracy in these TX-RX pairs, except the TX2-RX1 case. This is clearly the worst result for the MUSIC algorithm, as seen in Figure 9(c), and is most probably affected by harmful reflections from the stairs which are made of metal, concrete, and glass.

4.4.2. Case 2. Based on the results of Tables 7-9, the total RMSE, calculated over all TX cases, is $15.0^{\circ}$ for the PD, $10.2^{\circ}$ for the APPR algorithm, and $14.8^{\circ}$ for the MUSIC algorithm 

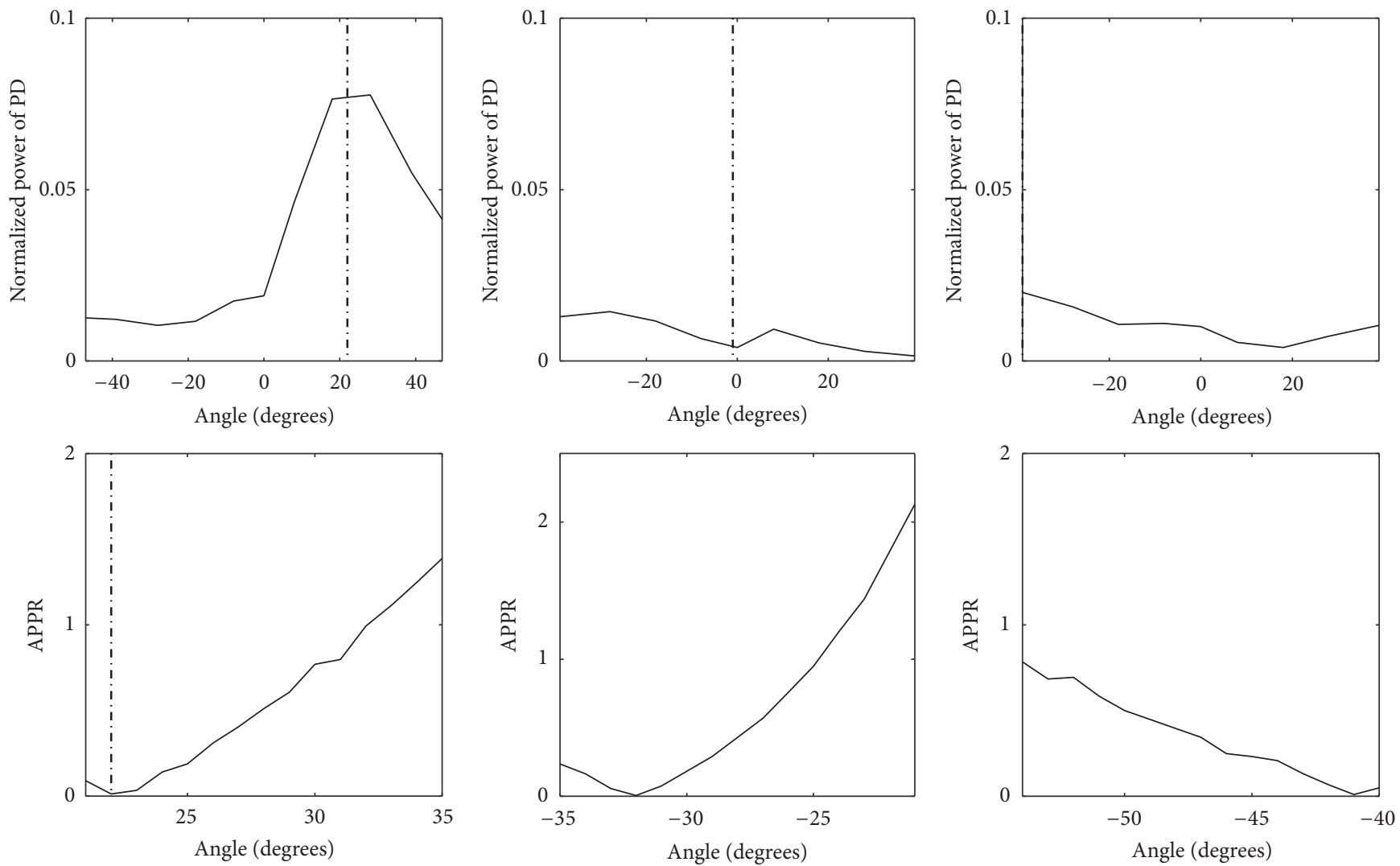

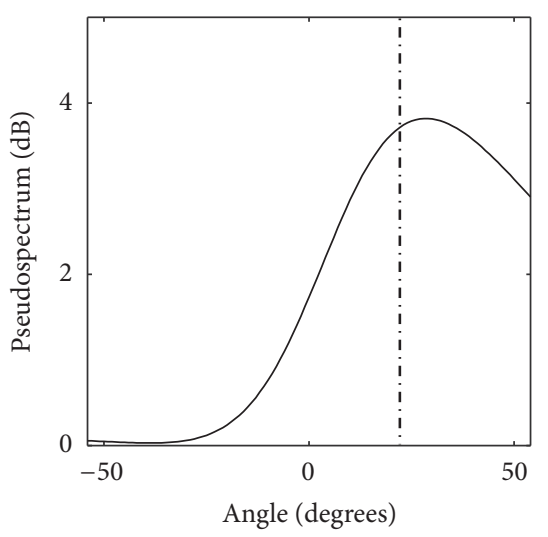

(a)

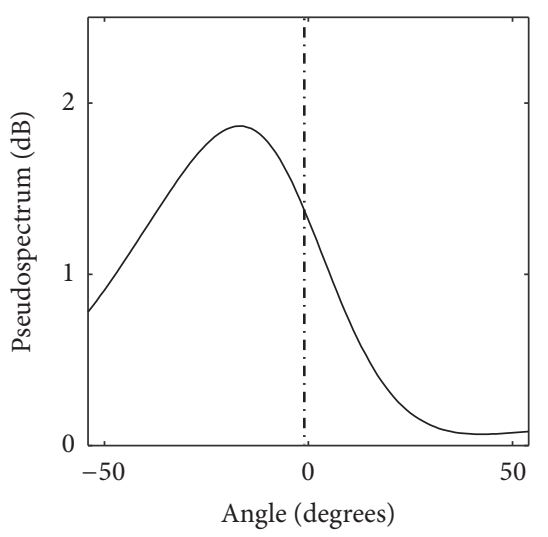

(b)

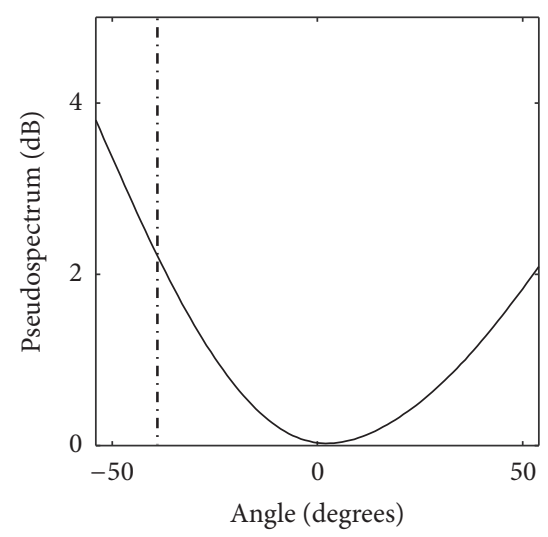

(c)

FIGURE 9: DoA estimation results with PD, APPR, and MUSIC algorithms in case 2 with different TX-RX pairs: (a) TX1-RX6 $\theta_{\text {real }}=22^{\circ}$, $(\mathrm{b})$ TX3-RX6 $\theta_{\text {real }}=-1^{\circ}$, and (c) TX2-RX1 $\theta_{\text {real }}=-39^{\circ}$.

in case 2. It can be seen that there is not a significant performance difference between case 1 and case 2 . From the tables, we can notice again that the estimation error is rather small, smaller than $11^{\circ}$ in many TX-RX pairs in several cases. However, there are also many larger DoA estimation errors which are most likely caused by severe reflections and other multipath effects, as explained earlier and illustrated in Figure 10(b).

4.4.3. Case 3. Based on the results of Tables 10-12, the total RMSE, calculated over all TX cases, is $14.1^{\circ}$ for the PD, $11.2^{\circ}$ for the APPR algorithm, and $32.1^{\circ}$ for the MUSIC algorithm in case 3 . It can be noticed that the difference of the performance is not big between cases 1-3 for the APPR algorithm. In particular, the difference of the RMSE is only $1.4^{\circ}$ between case 1 and case 3 . The results show that we can achieve almost the same performance using fewer radiation patterns; thus the overall signal storing time will be decreased. However, the adjacent radiation patterns cannot be too far away from each other, as seen in Section 4.1. Regarding the MUSIC algorithm, the RMSEs are significantly higher in case 3 when compared with cases $1-2$. We can conclude that the MUSIC algorithm does not work very well if the number of radiation patterns is only five in our 

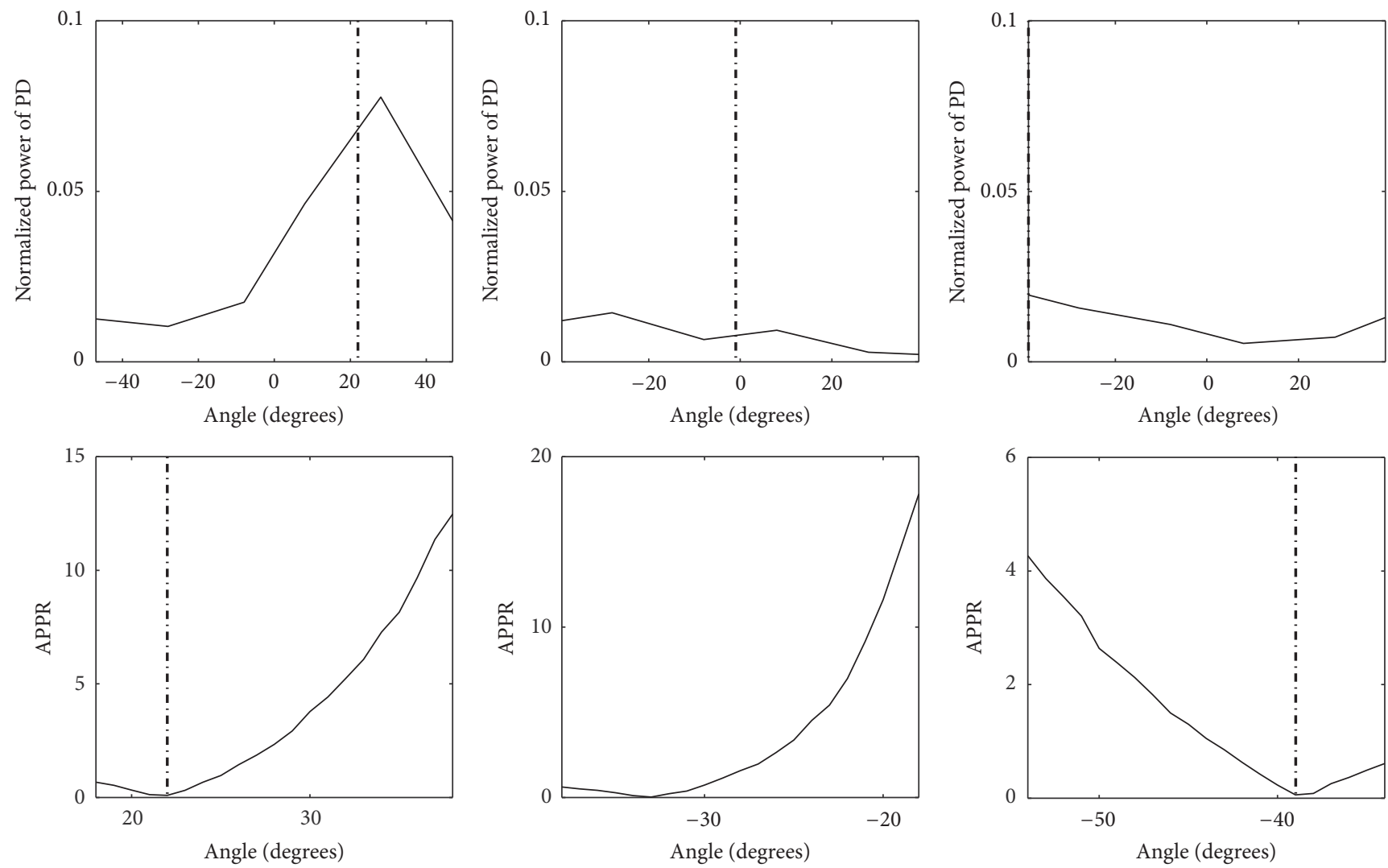

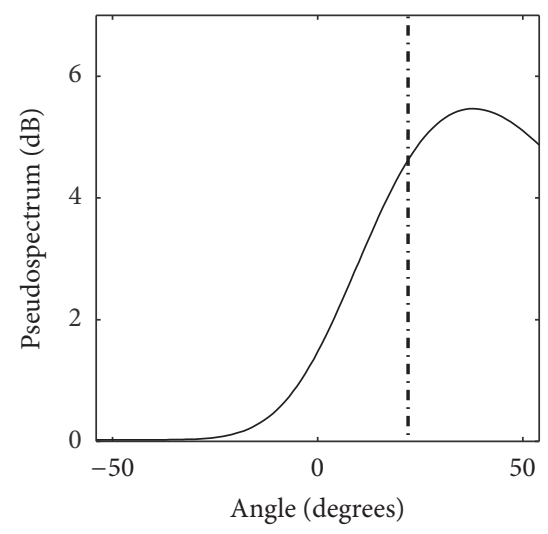

(a)

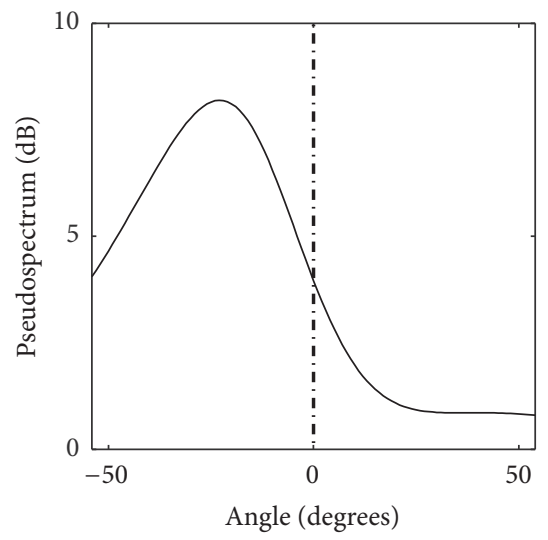

(b)

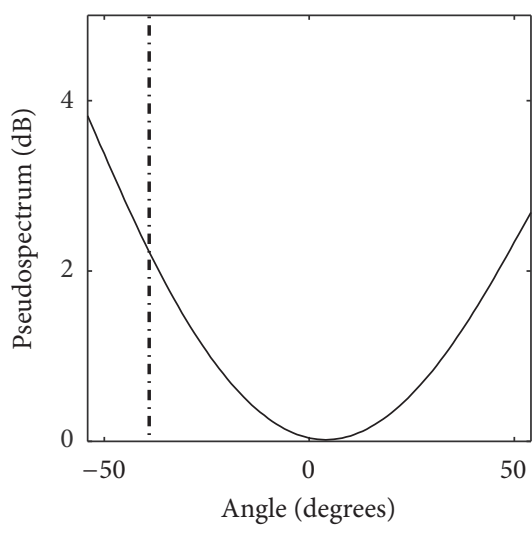

(c)

FIGURE 10: DoA estimation results with PD, APPR, and MUSIC algorithms in case 2 with different TX-RX pairs: $(\mathrm{a}) \mathrm{TX1}-\mathrm{RX} 6 \theta_{\text {real }}=22^{\circ},(\mathrm{b})$ TX3-RX6 $\theta_{\text {real }}=-1^{\circ}$, and (c) TX2-RX1 $\theta_{\text {real }}=-39^{\circ}$.

real world measurements. We can also notice that the total RMSE difference between case 1 and case 2 is only $1.2^{\circ}$ for the MUSIC algorithm. If fewer radiation patterns are selected, the complexity of the MUSIC algorithm will be reduced significantly, as explained in Section 4.2. To conclude, the selection of the number of radiation patterns is a tradeoff between the desired performance and computational complexity.

4.4.4. Discussion of the Experimental Results. Based on our observations from the experimental results, it seems that the APPR algorithm works better in a real multipath environment than the MUSIC algorithm. In our measurements, the measured signals experience both LoS and NLoS components due to severe multipath between the TX and the RX in the indoor environment. Due to the correlated multipath signals, the eigenvalue decomposition of the signal covariance cannot split the signal and noise subspaces; thus the RMSE increases significantly when compared with the AWGN channel simulations. In case 1, the total RMSE is $3.8^{\circ}$ less with the APPR algorithm than with the MUSIC algorithm. It can be concluded that the performance gap 

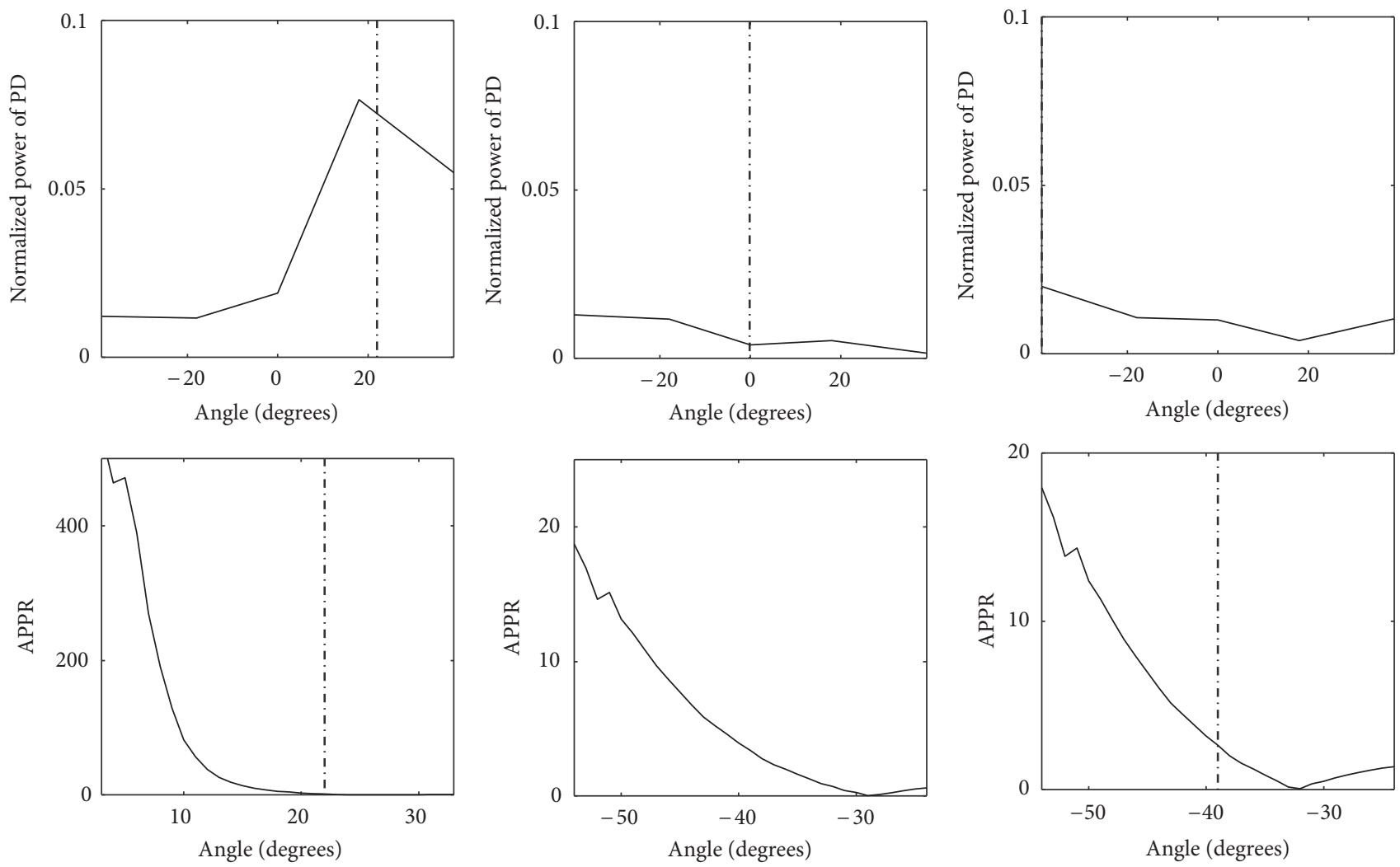

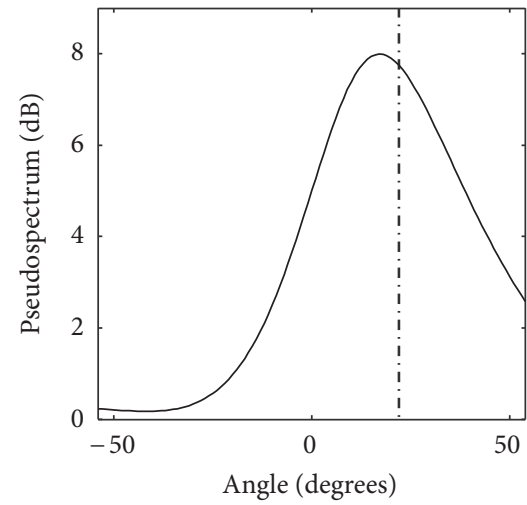

(a)

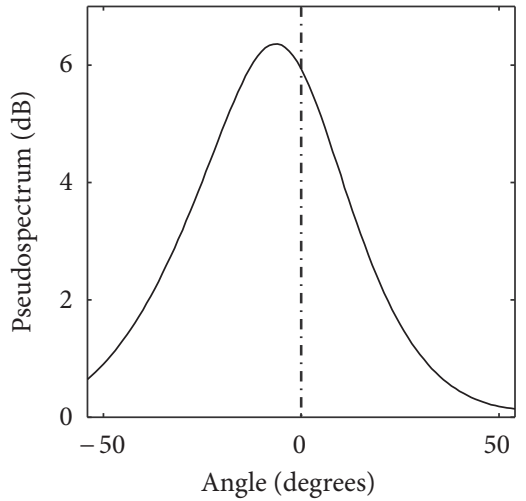

(b)

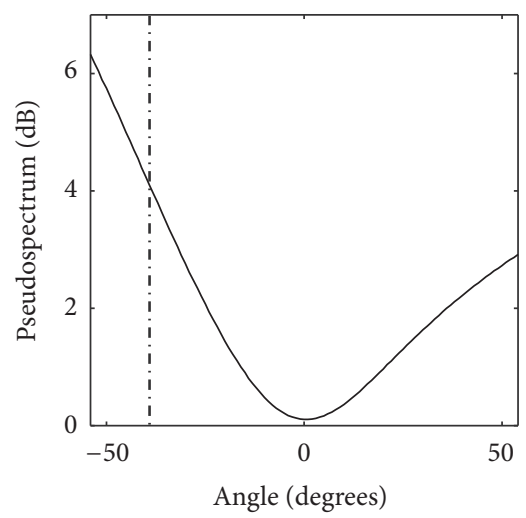

(c)

FIgURE 11: DoA estimation results with PD, APPR, and MUSIC algorithms in case 3 with different TX-RX pairs: (a) TX1-RX6 $\theta_{\text {real }}=22^{\circ}$, (b) TX3-RX6 $\theta_{\text {real }}=-1^{\circ}$, and (c) TX2-RX1 $\theta_{\text {real }}=-39^{\circ}$.

between these two algorithms increases when the number of radiation patterns is decreased.

The resolution of both the algorithms is the same, that is, $1^{\circ}$. However, the computational complexity of the algorithms is significantly different, as explained in Section 4.2. The APPR algorithm has clearly lower computational complexity than MUSIC algorithm because the algorithm does not use eigenvalue decomposition. We can conclude that both algorithms, especially the APPR algorithm, and the considered LWA structure enable reliable DoA estimation, despite very challenging demonstration and measurement environment.
In our measurement, we have assumed, particularly in the MUSIC algorithm case, that only one signal is received in each receiver. We have noticed that there are multiple peaks in the PD plots. It is clear that multipath or other signals result in additional peaks in the figures. These interference signals are one reason why the performance of the MUSIC algorithm is much poorer than in AWGN simulations. In general, signals are better resolvable if the bandwidth is increased (e.g., using impulse radio [24]) and if the beamwidth of the antenna is decreased. In literature, Akaiake information criterion (AIC) [25] is presented and the minimum description length (MDL) [26] algorithms estimate the number of the incident signals. 
However, it is shown that these algorithms can estimate a wrong number of components for a small sample size and a low SNR [27]. In future work, we will study how we could resolve the received signals, that is, multipath and interference signals, and estimate the number of the received signals reliably so that the DoA estimation algorithms can work in a more robust way.

\section{Conclusion}

In this paper, we considered DoA estimation with a certain type of reconfigurable antennas, namely, CRLH-LWAs. We started by presenting the APPR algorithm for two-port LWAs. Thereafter, we evaluated the performance of the APPR solution by numerical simulations in an AWGN channel and with varying numbers of selected radiation patterns. The results were also compared with those of the LWA-based MUSIC algorithm. We continued by evaluating the DoA estimation performance of both methods in an indoor environment based on real world measurements involving typical multipath propagation with both line-of-sight and nonline-of-sight components. Not only the results of numerical simulations but also the measurement-based results showed that the DoA estimates were in a good agreement with the real DoAs, especially with the APPR method, indicating that CRLH-LWAs are capable of successful DoA estimation while having often a smaller form factor than conventional antenna arrays with multiple antenna elements. To conclude, the combination of the proposed DoA estimation algorithms and the CRLH-LWA implementation can provide a valuable solution for future generation wireless communications systems where spectrum reuse, interference avoidance, and device localization are of special interest.

\section{Conflicts of Interest}

The authors declare that they have no conflicts of interest.

\section{Acknowledgments}

This work has been performed in the framework of the Reconfigurable Antenna Based Enhancement of Dynamic Spectrum Access Algorithms (READS) and Future SmallCell Networks using Reconfigurable Antennas (FUNERA) projects which were funded by VTT Technical Research Center of Finland and the Finnish Funding Agency for Technology and Innovation (Tekes). The authors would like to thank Adant S.r.l. for the antenna prototypes. The measurements have been carried out at the Drexel University. This work was also supported by CNS-1147838 from the U.S. National Science Foundation as part of the Wireless Innovation between Finland and United States (WiFiUS) partnership.

\section{References}

[1] J. D. Kraus and R. J. Marhefka, Antennas for All Applications, McGraw-Hill, Boston, MA, USA, 3rd edition, 2001.
[2] C. A. Balanis, Antenna Theory: Analysis and Design, John Wiley Sons, Hoboken, NJ, 3rd edition, 2005.

[3] T. Ohira and K. Gyoda, "Electronically steerable passive array radiator antennas for low-cost analog adaptive beamforming," in Proceedings of the 2000 IEEE International Conference on Phased Array Systems and Technology, pp. 101-104, May 2011.

[4] J. R. De Luis and F. De Flaviis, "A reconfigurable dual frequency switched beam antenna array and phase shifter using PIN diodes," in Proceedings of the 2009 IEEE International Symposium on Antennas and Propagation and USNC/URSI National Radio Science Meeting, APSURSI 2009, June 2009.

[5] C. Caloz and T. Itoh, Electromagnetic Metamaterials Transmission Line Theory and Microwave Applications, John Wiley Sons, Hoboken, NJ, 2006.

[6] R. Schmidt, "Multiple emitter location and signal parameter estimation," in Proceedings of the RADC Spectrum Estimation Workshop, pp. 243-258, 1979.

[7] K.-C. Huarng and C.-C. Yeh, "A Unitary Transformation Method for Angle-of-Arrival Estimation," IEEE Transactions on Signal Processing, vol. 39, no. 4, pp. 975-977, 1991.

[8] M. D. Zoltowski, G. M. Kautz, and S. D. Silverstein, "Beamspace root-MUSIC," IEEE Transactions on Signal Processing, vol. 41, no. 1, pp. 344-364, 1993.

[9] M. Haardt and J. A. Nossek, "Unitary ESPRIT: how to obtain increased estimation accuracy with a reduced computational burden," IEEE Transactions on Signal Processing, vol. 43, no. 5, pp. 1232-1242, 1995.

[10] R. Roy, ESPRIT: Estimation of Signal Parameters via Rotational Invariance Techniques [Phd. Dissertation], Stanford University, Stanford, CA, USA.

[11] S. Sugiura, "A review of recent patents on reactance-loaded reconfigurable antennas," Recent Patents on Electrical Engineering, vol. 2, no. 3, pp. 200-206, 2009.

[12] H. Paaso, A. Mammela, D. Patron, and K. R. Dandekar, "Modified MUSIC algorithm for doa estimation using CRLH leakywave antennas," in Proceedings of the 2013 8th International Conference on Cognitive Radio Oriented Wireless Networks and Communications, CROWNCOM 2013, pp. 166-171, usa, July 2013.

[13] D. Patron, H. Paaso, A. Mammela, D. Piazza, and K. R. Dandekar, "Improved design of a CRLH leaky-wave antenna and its application for DoA estimation," in Proceedings of the 2013 3rd IEEE-APS Topical Conference on Antennas and Propagation in Wireless Communications, IEEE APWC 2013, pp. 1343-1346, September 2013.

[14] H. Paaso, N. Gulati, D. Patron et al., "DoA estimation using compact CRLH leaky-wave antennas: novel algorithms and measured performance," IEEE Transactions on Antennas, In press.

[15] V. Vakilian, J.-F. Frigon, and S. Roy, "Direction-of-arrival estimation in a clustered channel model," in Proceedings of the 2012 IEEE 10th International New Circuits and Systems Conference, NEWCAS 2012, pp. 313-316, can, June 2012.

[16] V. Vakilian, H. V. Nguyen, S. Abielmona, S. Roy, and J.-F. Frigon, "Experimental study of direction-of-arrival estimation using reconfigurable antennas," in Proceedings of the 2014 IEEE 27th Canadian Conference on Electrical and Computer Engineering, CCECE 2014, can, May 2014.

[17] C. Plapous, J. Cheng, E. Taillefer, A. Hirata, and T. Ohira, "Reactance domain MUSIC algorithm for ESPAR antennas," in Proceedings of the 33rd European Microwave Conference, EuMC 2003, pp. 793-796, deu, October 2003. 
[18] S. Akkar and A. Gharsallah, "Reactance domains unitary MUSIC algorithms based on real-valued orthogonal decomposition for electronically steerable parasitic array radiator antennas," IET Microwaves, Antennas and Propagation, vol. 6, no. 2, pp. 223-230, 2012.

[19] Y. Ozaki, J. Ozawa, E. Taillefer, J. Cheng, and Y. Watanabe, "A simple DoA estimator using adjacent pattern power ratio with switched beam antenna," Progress In Electromagnetics Research C, vol. 22, pp. 55-71, 2011.

[20] M. Wax, Detection and estimation superimposed signals [Ph.D. thesis], Stanford University, Stanford, 1985.

[21] Z. Chen, Introduction to Direction-of-Arrival Estimation, Artech House Books, Norwood, MA, USA, 2010.

[22] P. Pirsch, Architectures for Digital Signal Processing, Artech House Books, John Wiley \& Sons, Chichester, UK, 1996.

[23] "Mango communications WARP v3 kit," http://mangocomm .com/products/kits/warp-v3-kit.

[24] S. Gezici, Z. Tian, G. B. Giannakis et al., "Localization via ultrawideband radios: a look at positioning aspects of future sensor networks," IEEE Signal Processing Magazine, vol. 22, no. 4, pp. 70-84, 2005.

[25] H. Akaike, "A new look at the statistical model identification," IEEE Transactions on Automatic Control, vol. 19, no. 6, pp. 716723, 1974.

[26] M. Wax and T. Kailath, "Detection of signals by information theoretic criteria," Institute of Electrical and Electronics Engineers. Transactions on Acoustics, Speech, and Signal Processing, vol. 33, no. 2, pp. 387-392, 1985.

[27] P. M. Djuric, "A model selection rule for sinusoids in white gaussian noise," IEEE Transactions on Signal Processing, vol. 44, no. 7, pp. 1744-1751, 1996. 


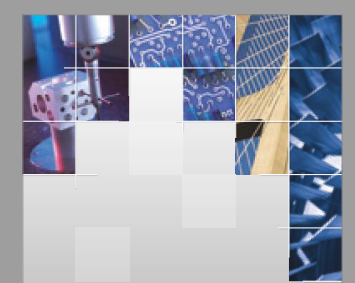

\section{Enfincering}
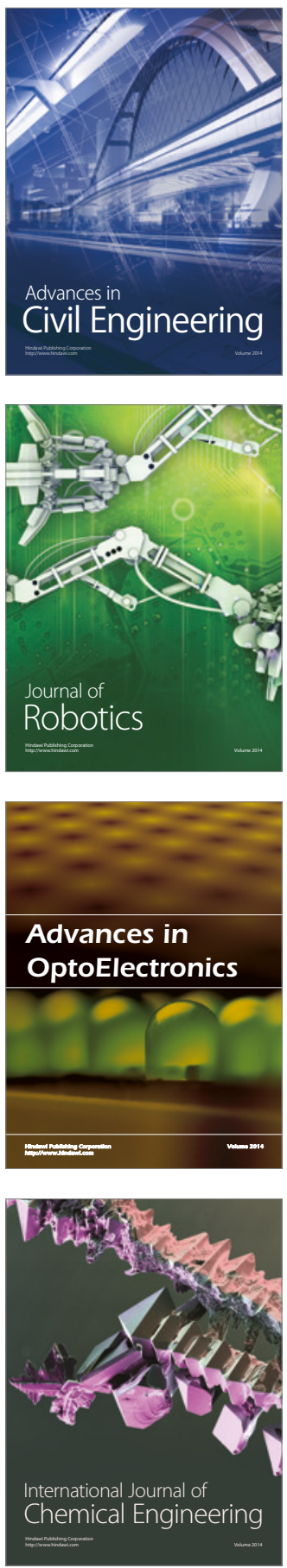

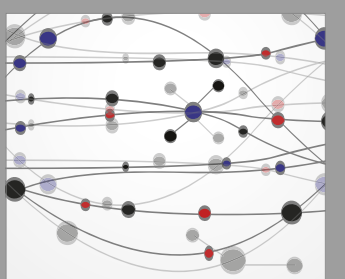

The Scientific World Journal

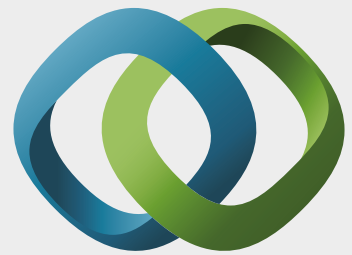

\section{Hindawi}

Submit your manuscripts at

https://www.hindawi.com
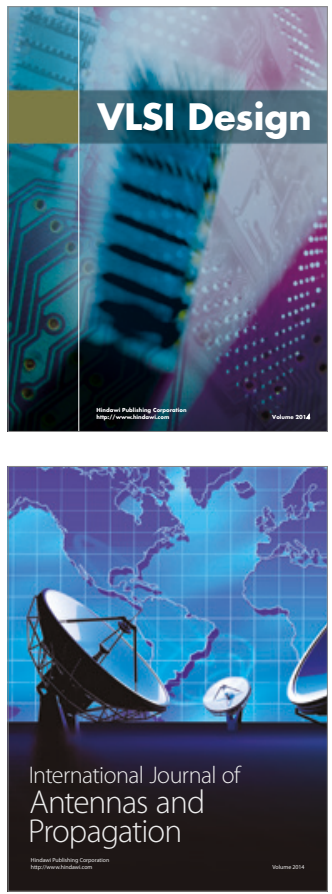

\section{Rotating}

Machinery
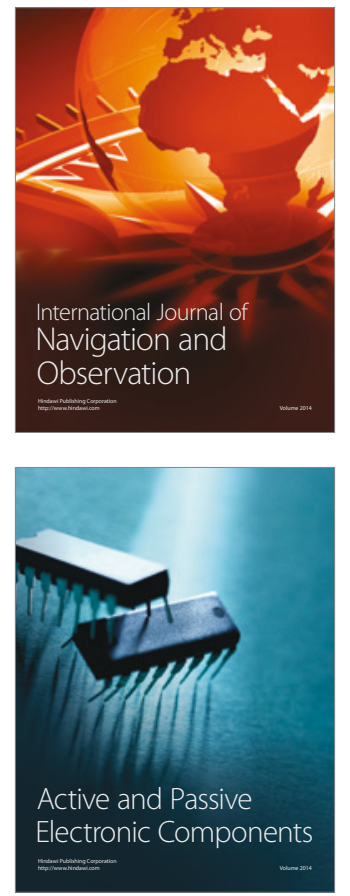
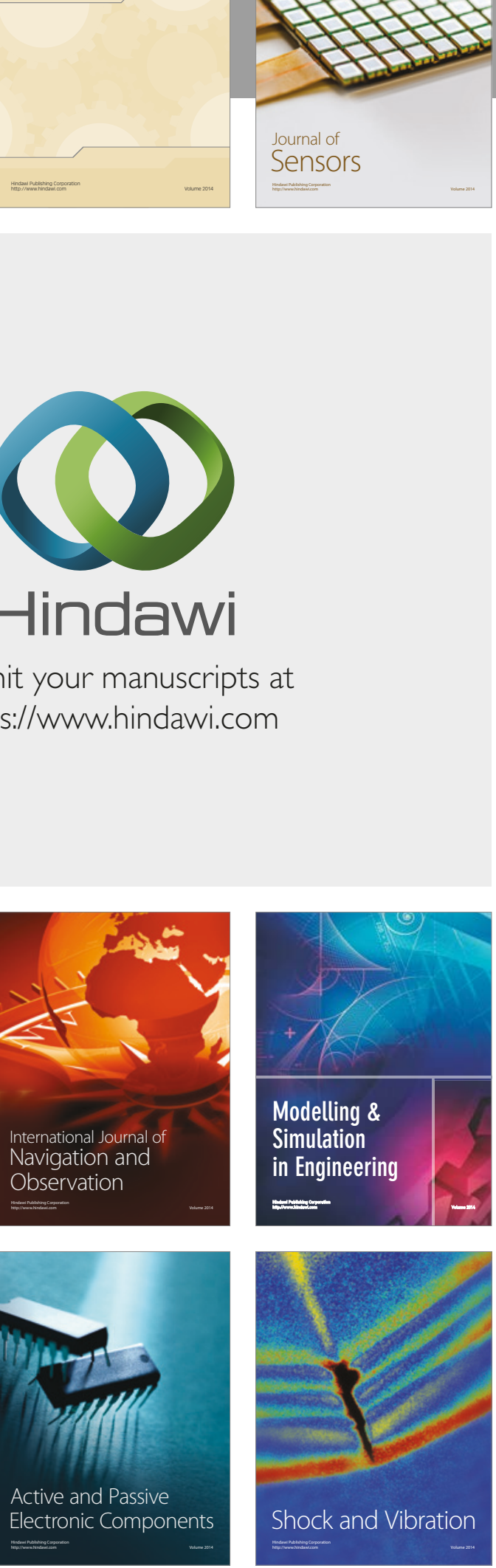
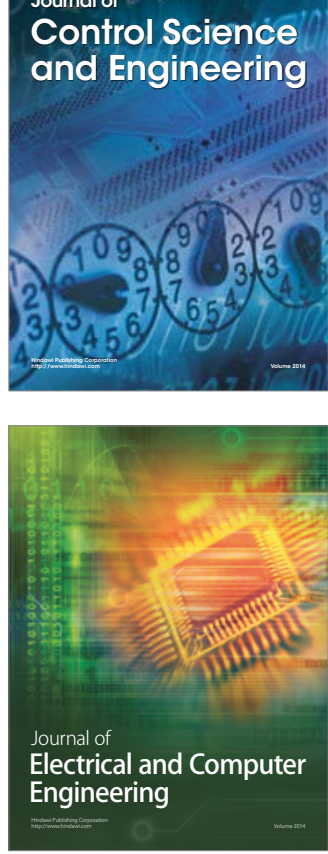

Distributed

Journal of

Control Science

and Engineering
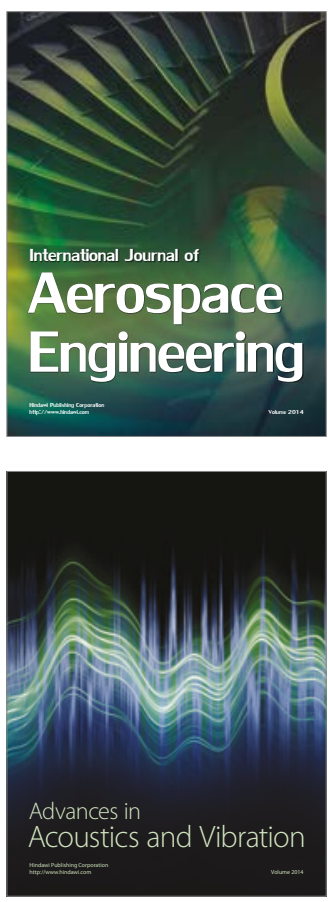

Sensor Networks 